University of South Florida

DIGITAL COMMONS

Digital Commons @ University of

@ UNIVERSITY OF SOUTH FLORIDA

South Florida

Marine Science Faculty Publications

College of Marine Science

2020

\title{
Revealing the Intensity of Turbulent Energy Transfer in Planetary Atmospheres
}

\author{
Simon Cabanes \\ Sapienza Universitá di Roma \\ Stefania Espa \\ Sapienza Universitá di Roma \\ Boris Galperin \\ University of South Florida, bgalperin@usf.edu \\ Roland M. Young \\ UAE University \\ Peter L. Read \\ University of Oxford
}

Follow this and additional works at: https://digitalcommons.usf.edu/msc_facpub

Part of the Life Sciences Commons

\section{Scholar Commons Citation}

Cabanes, Simon; Espa, Stefania; Galperin, Boris; Young, Roland M.; and Read, Peter L., "Revealing the Intensity of Turbulent Energy Transfer in Planetary Atmospheres" (2020). Marine Science Faculty Publications. 1449.

https://digitalcommons.usf.edu/msc_facpub/1449

This Article is brought to you for free and open access by the College of Marine Science at Digital Commons @ University of South Florida. It has been accepted for inclusion in Marine Science Faculty Publications by an authorized administrator of Digital Commons @ University of South Florida. For more information, please contact digitalcommons@usf.edu. 


\section{Geophysical Research Letters}

\author{
RESEARCH LETTER \\ 10.1029/2020GL088685 \\ Key Points: \\ - We present a new and universal \\ diagnostic to reveal the intensity \\ of turbulent energy transfer in \\ planetary atmospheres \\ - We show that turbulent energy \\ transfer in Saturn's atmosphere is 4 \\ times less intense than Jupiter's
}

Supporting Information:

- Supporting Information S1

Correspondence to:

S. Cabanes,

cabanes.simon@gmail.com

Citation:

Cabanes, S., Espa, S., Galperin, B., Young, R. M. B., \& Read, P. L. (2020). Revealing the intensity of turbulent energy transfer in planetary atmospheres. Geophysical Research Letters, 47, e2020GL088685. https:// doi.org/10.1029/2020GL088685

Received 9 MAY 2020

Accepted 30 OCT 2020

Accepted article online 16 NOV 2020

(C)2020. American Geophysical Union. All Rights Reserved.

\section{Revealing the Intensity of Turbulent Energy Transfer in Planetary Atmospheres}

\author{
Simon Cabanes ${ }^{1}$ (D) , Stefania Espa ${ }^{1}$ (D), Boris Galperin ${ }^{2}$ iD), Roland M. B. Young ${ }^{3}$ (D), \\ and Peter L. Read ${ }^{4}$ iD \\ ${ }^{1}$ DICEA, Sapienza Universitá di Roma, Rome, Italy, ${ }^{2}$ College of Marine Science, University of South Florida, St. \\ Petersburg, FL, USA, ${ }^{3}$ Department of Physics and National Space Science and Technology Center, UAE University, Al \\ Ain, United Arab Emirates, ${ }^{4}$ Atmospheric, Oceanic and Planetary Physics, Department of Physics, University of Oxford, \\ Oxford, UK
}

\begin{abstract}
Images of the giant planets Jupiter and Saturn show highly turbulent storms and swirling clouds that reflect the intensity of turbulence in their atmospheres. Quantifying planetary turbulence is inaccessible to conventional tools, however, since they require large quantities of spatially and temporally resolved data. Here we show, using experiments, observations, and simulations, that potential vorticity (PV) is a straightforward and universal diagnostic that can be used to estimate turbulent energy transfer in a stably stratified atmosphere. We use the conservation of PV to define a length scale, $L_{M}$, representing a typical distance over which PV is mixed by planetary turbulence. $L_{M}$ increases as the turbulent intensity increases and can be estimated from any latitudinal PV profile. Using this principle, we estimate $L_{M}$ within Jupiter's and Saturn's tropospheres, showing for the first time that turbulent energy transfer in Saturn's atmosphere is four times less intense than Jupiter's.
\end{abstract}

\section{Introduction}

In planetary atmospheres, it is common that the outer atmospheric envelope contains highly turbulent flows powered by solar energy and by a heat flux from within the planet itself (Ingersoll \& Pollard, 1982; Vallis, 2006). These thermal energy sources transform into sources of atmospheric motion by driving turbulent eddies whose typical scales do not exceed the Rossby deformation radius, which is typically $\sim 2,500 \mathrm{~km}$ for both Jupiter (Young \& Read, 2017) and Saturn (Read et al., 2009). It ensures the growth of powerful large scale zonal jets (east-west directed flow with 10,000-20,000 km latitudinal scale) and a host of waves and vortices, among which the small scale forcing is unobservable by direct measurement.

Planetary turbulence is characterized by the nonlinear transfer of energy between different scales of motions in processes known as cascades. Because rotation inhibits vertical motion, turbulent planetary flows are quasi-two-dimensional in the horizontal (i.e., in latitude-longitude) and these cascades transfer energy upscale, from small-scale energy sources up to the large-scale jets, resulting in a kinetic energy spectrum that accords with the well-known Kolmogorov-Kraichnan (KK) law $\Pi_{\epsilon}^{2 / 3} n^{-5 / 3}$ (Kraichnan, 1967). In KK's cascade, the energy transfer rate, $\Pi_{\epsilon}$, is crucial for the understanding and quantification of planetary turbulence. It uniquely describes a global estimate of the power continuously exchanged between all scales of motions, that is, between jets, waves, and eddies.

To measure $\Pi_{\epsilon}$ in planetary flows, a prerequisite is to collect two-dimensional (2-D) horizontal high-resolution velocity maps and then either compute a spectral decomposition or use methods based on structure functions (Arbic et al., 2014). This has been done for several numerical and laboratory experiments that emulate planetary-like flows (Augier \& Lindborg, 2013; Cabanes et al., 2020; Read et al., 2018; Schneider $\&$ Liu, 2009). Yet for real planets it is only recently that the Cassini mission, by taking high-resolution images of Jupiter's cloud deck, has allowed the first global estimates of the power transferred from small-scale forcing to the jets in a gas giant's atmosphere to be made, yielding $10^{-5} \leq \Pi_{\epsilon} \leq 10^{-4} \mathrm{~W} \mathrm{~kg}^{-1}$ (Galperin, Young, et al., 2014; Young \& Read, 2017). However, due to a lack of appropriate imaging data, the methods used to make this estimate are impractical for other planets, such as Saturn, Uranus, and Neptune, and recently discovered exoplanets, such as gas dwarf planets and hot Jupiters. Here, we propose a new method that can be used to quantify this power $\Pi_{\epsilon}$ using only a limited number of readily available measurements. 


\section{Potential Vorticity and Its Link to $\Pi_{\epsilon}$}

It has long been known that planetary rotation and stable stratification facilitate the material conservation of potential vorticity (PV) (Pedlosky, 2013). PV is intimately related to the Rossby waves that emerge due to a gradient of planetary vorticity, or the $\beta$-effect (Rhines, 1975; Vallis \& Maltrud, 1993). $\beta=(2 \Omega / R) \cos \theta$, where $R$ is the planetary radius, $\Omega$ is the rotation rate, and $\theta$ is latitude. In its simplest, incompressible form,

$$
P V=(\zeta+2 \Omega) / H
$$

PV combines a dynamical term, the vertical component of relative vorticity $\zeta$, with the intrinsic planetary parameters $\Omega$ and the fluid depth $H$. In planetary atmospheres, turbulent mixing, characterized by the relative vorticity $\zeta$, causes parallel bands of constant PV to emerge, and these bands result in multiple zonal jets (Cho \& Polvani, 1996; Dritschel \& McIntyre, 2008; Marcus \& Lee, 1998; Marcus \& Shetty, 2011). PV banding in latitude is thought to result from breaking Rossby waves producing turbulent mixing, which leads to the local homogenization of PV (Dritschel \& McIntyre, 2008; Marcus \& Shetty, 2011; Phillips, 1956). In this framework, "there is no turbulence without waves" (Galperin, Hoemann, et al., 2014), and Rossby waves "conspire" with quasi-2-D turbulence to form jets (Dritschel \& McIntyre, 2008). In the present study, we turn this notion on its head, arguing that turbulence generate waves and that PV mixing characterizes the global power $\Pi_{\epsilon}$ associated with turbulence, including Rossby wave turbulence together with small-scale energetic forcing.

Here, we aim to establish the relationship that exists between PV mixing and the turbulent power $\Pi_{\epsilon}$. To do so, we make use of the analogy drawn by Dritschel and McIntyre (2008) between the banding of constant PV in planetary atmospheres and the layering of constant density in the oceans caused by the turbulent mixing of the vertical density gradient. In the oceans, Thorpe (2005) showed that the vertical density profile is made nonmonotonic by turbulent motion that carries more dense, heavier water above lighter water over a typical distance called the Thorpe scale $L_{T}$. The Thorpe scale is estimated by a "sorting algorithm" that converts an unstable, nonmonotonic density profile into a stably stratified profile with density increasing downward (Thorpe, 2005). The Thorpe scale is approximately equal to the Ozmidov scale $L_{O}=\left(\Pi_{\varepsilon} / N^{3}\right)^{1 / 2}$, where $\Pi_{\varepsilon}$ and $N$ are the rate of turbulent energy transfers to dissipation and the Brunt-Väisälä frequency, respectively. Physically, $L_{O}$ is a scale at which the turbulent eddy turnover time is equal to the period of internal gravity waves. Numerous experiments and observations show that $0.25 L_{O} \leq L_{T} \leq 4 L_{O}$ (Thorpe, 2005).

The Thorpe scale can often be easily computed and has become a widely used measure to estimate the rate of turbulent energy transfer to dissipation, $\Pi_{\varepsilon}$, in stably stratified flows in the ocean (Gargett \& Garner, 2008; Thorpe, 2005) and atmosphere (Clayson \& Kantha, 2008; Gavrilov et al., 2005; Kantha \& Hocking, 2011), and in computer simulations (Klymak \& Legg, 2010). Here we extend the analogy between PV banding in latitude and density layering in the vertical by adapting Thorpe's sorting algorithm to monotonize latitudinal PV profiles. This introduces an analog of the Thorpe scale, which we denote $L_{M}$, that leads to an estimate of the turbulent power $\Pi_{\epsilon}$ in giant planet atmospheres. By applying Thorpe's sorting algorithm to PV monotonization, we explore the analogy between vertical and horizontal turbulent mixing in, respectively, stably stratified and quasi-geostrophic (QG) flows.

To complete our approach, we need an analog for $L_{O}$ in planetary turbulence. We suggest the length scale $L_{\beta} \approx\left(\Pi_{\epsilon} / \beta^{3}\right)^{1 / 5}$, which compares, by analogy with $L_{O}$, the turbulent power $\Pi_{\epsilon}$ with the strength of the background planetary vorticity $\beta$ (Vallis \& Maltrud, 1993). The question is whether there is a universal relationship between $L_{M}$ and $L_{\beta}$. To address this, we extend the work of Galperin, Hoemann, et al. (2014), in which $L_{M}$ and $L_{\beta}$ were estimated from a limited set of laboratory measurements, by using an unprecedented combination of three independent data sets including laboratory experiments, direct observations of Jupiter atmospheric dynamics, and a numerical model of Saturn's general circulation. This data set allows us to compute both the spectral analysis of 2-D velocity fields, necessary to estimate $L_{\beta}$, and the monotonization of instantaneous PV profiles, necessary to estimate $L_{M}$. Then, we make use of our new diagnostic based on PV to give the first estimate of the global turbulent power $\Pi_{\epsilon}$ from direct observations of Saturn's atmospheric dynamics.

\section{Three Independent Sources of Zonal Jet Spectral Diagnostics}

The first source used to obtain zonal jet spectral diagnostics is an experimental device that reproduces the conditions required to generate planetary-like zonal jets. The experimental setup is a rotating $70 \mathrm{~cm}$ square 

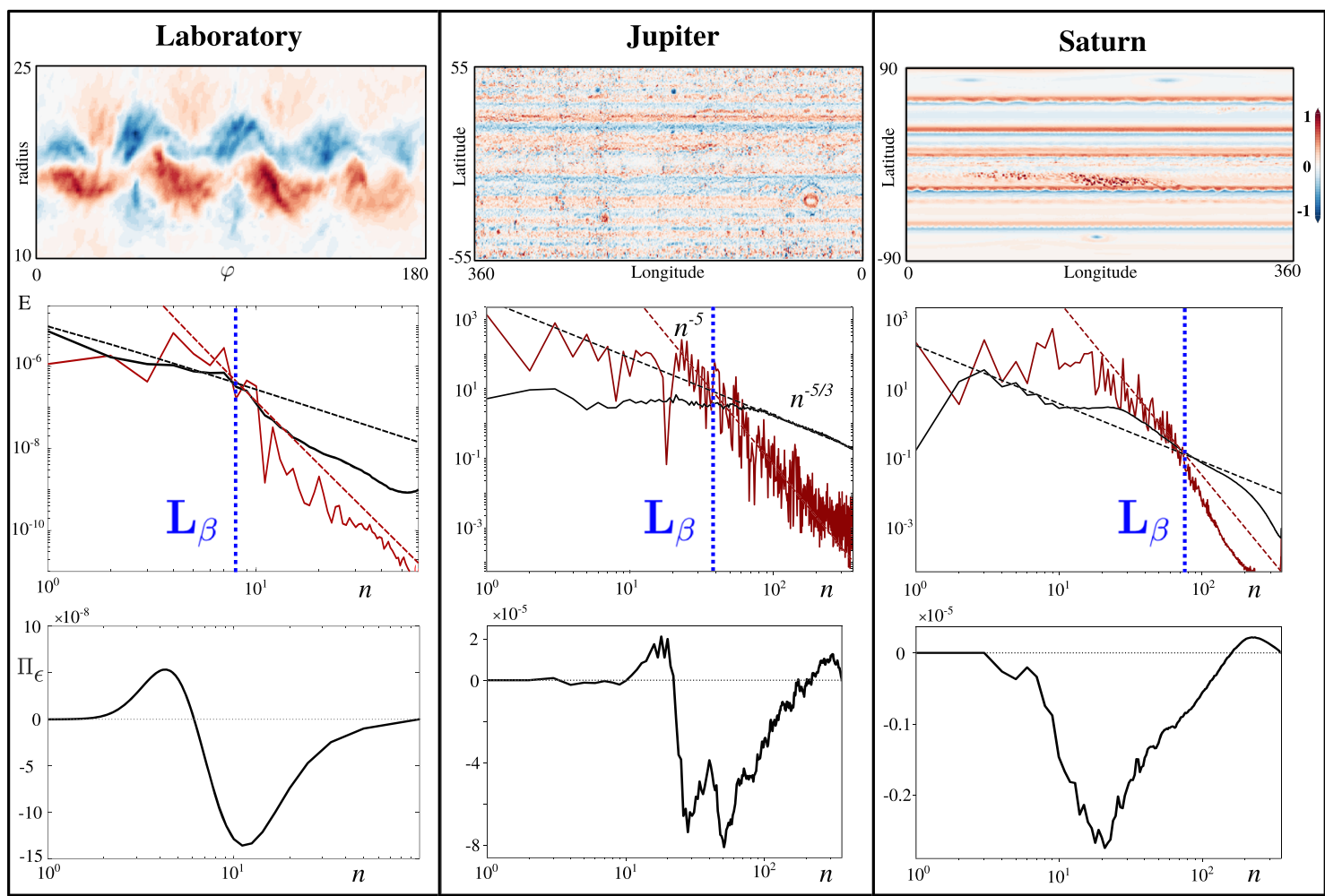

Figure 1. Relative vorticity horizontal maps, energy spectra and energy fluxes. Top panels: Vertical component of the relative vorticity $\zeta$ in $\mathrm{s}^{-1}$. The color bar is stretched by a factor of 2 for the $180^{\circ}$ westward laboratory jet (3: westward jet $180^{\circ}$ ), by $\times 10^{-4}$ for the G14g data computed from Cassini images of Jupiter, and by $4 \times 10^{-5}$ for the Saturn GCM. Middle panels: Zonal energy spectra $E_{Z}(n)$ (red) and velocity fluctuations energy spectra $E_{K K}(n)\left(\right.$ black) in m ${ }^{-2} \mathrm{~s}^{-2}$ as a function of nondimensional radial/latitudinal wavenumber $n$. The dashed lines are theoretical predictions of the zonal energy $E_{Z}(n)=0.2 \beta^{2} n^{-5}($ red) and the Kolmogorov-Kraichnan (KK) energy $E_{K K}(n)=6 \Pi_{\epsilon}^{2 / 3} n^{-5 / 3}$. In the laboratory $\beta \simeq 53 \mathrm{~m}^{-1} \mathrm{~s}^{-1}$, for Jupiter $\beta \simeq 2.5 \times 10^{-12} \mathrm{~m}^{-1} \mathrm{~s}^{-1}$, and for Saturn $\beta \simeq 2.83 \times 10^{-12} \mathrm{~m}^{-1} \mathrm{~s}^{-1}$. Vertical lines are transitional scales $L_{\beta}$ that correspond to the intersection of the zonal and KK theoretical spectra. Bottom panels: energy fluxes $\Pi_{\epsilon}$ in $\mathrm{W} \mathrm{kg}^{-1}$ as a function of nondimensional radial/latitudinal wavenumber $n$. Positive/negative values of the fluxes refer to downscale/upscale energy transfers. Energy spectra and fluxes are computed from instantaneous velocity maps at steady state and averaged in time (over 58 rotation periods in the laboratory, 4 days for Jupiter observations, and the two last simulated years for Saturn GCM) at each mode $n$.

tank, filled with $4 \mathrm{~cm}$ depth of salt water, which is spun at 29 revolutions per minute. We ran nine independent experiments that generated zonal jets via electromagnetic forcing, using a range of circular magnets placed at the bottom of the tank and centered on the rotating spin axis along an arc of either $90^{\circ}$ or $180^{\circ}$ (see the experimental device in the supporting information). Experiments differed by the strength and the direction of the forcing, investigating both eastward and westward jets (details are given in supporting information section S1). Experimental measurements of the surface velocities were acquired by recording the tracks of small floating particles from an overhead camera centered in the rotating frame and then by analyzing their paths using a Lagrangian tracking method (Galperin et al., 2016). The 2-D surface velocity maps obtained for each configuration spanned 58 rotation periods (two minutes) at a frequency of $20 \mathrm{~Hz}$ (see field maps in Figure 1 and supporting information).

The second approach is to use maps of Jupiter's observed cloud-top winds. Two-dimensional horizontal velocity maps were obtained in Galperin, Young, et al. (2014) using cloud tracking of high-resolution images taken during Cassini's flyby of Jupiter (see zonal velocity map in Figure 1 and supporting information). In planetary science, PV has long been considered to be fundamentally important when investigating atmospheric dynamics. Therefore, we also make use of zonally averaged Jovian PV profiles computed in Read et al. (2006), at different atmospheric pressure levels (see supporting information figures and section S1). In atmospheric flows assumed to be adiabatic and frictionless, various materially conserved PV diagnostics can be derived. The most fundamental form is the Ertel PV formulation on isentropic surfaces, usually called IPV (Ertel \& Rossby, 1949). Under the QG approximation, an alternative form of PV defined on isobaric surfaces is QGPV (Gierasch et al., 2004). Both IPV and QGPV involve thermodynamic terms, in contrast to the barotropic PV in Equation 1, and their exact formulations are detailed in supporting information section S1. 
Finally, we use numerical simulations and observations to obtain the same diagnostics for Saturn. Read et al. (2009) repeated the same procedure as for Jupiter and derived IPV and QGPV zonally averaged profiles in latitude at various pressure levels (see all profiles in the supporting information). Unlike Jupiter, however, current observations of Saturn lack the global high-resolution images required to reconstruct 2-D horizontal wind maps. As a result, PV monotonization is the only way we can diagnose the power in Saturn's observed turbulent flow. Using numerical simulations, however, we can obtain 2-D horizontal wind maps. To ensure that our representation of Saturn's dynamics is as realistic as possible, we compute a spectral analysis and PV monotonization using data from a $0.5^{\circ}$ resolution, multiannual 3-D numerical simulation obtained by the Saturn Global Climate Model (GCM) described by Spiga et al. (2020) and (Cabanes et al., 2020). This GCM is designed to explore Saturn's tropospheric and stratospheric dynamics with a new icosahedral dynamical core DYNAMICO (Dubos et al., 2015) and realistic radiative transfer (Guerlet et al., 2014). Characteristic 2-D horizontal velocity maps are shown in Figure 1 and supporting information figures. We perform our spectral analysis and PV monotonization within the upper troposphere and lower stratosphere, corresponding to $2 \leq p \leq 650 \mathrm{hPa}$.

\section{Turbulent Power Computed From the Three Types of Zonal Jets}

Figure 1 shows 2-D maps of the instantaneous relative vorticity from a laboratory jet, Jupiter observations, and the Saturn GCM. We compute kinetic energy spectra of the velocity maps by using a Bessel-Fourier decomposition in the cylindrical geometry of the laboratory experiment and a spherical harmonic decomposition in the spherical geometry of Jupiter and Saturn (see supporting information section S1). In order to better characterize the nonlinear dynamics of eddy-eddy interactions, we also compute the fluxes of kinetic energy between different scales of motions, using a filtering procedure in the laboratory (Boffetta \& Musacchio, 2010; Chen et al., 2006) and the spherical harmonic decomposition (Augier \& Lindborg, 2013; Boer, 1983) for Jupiter observations and Saturn GCM (see details in supporting information section S1). The spectra and energy fluxes are also shown in Figure 1.

In all cases, we can fit kinetic energy spectra with the theoretical anisotropic zonal flow spectrum:

$$
E_{Z}(n)=C_{Z} \beta^{2} n^{-5}
$$

and the KK-law spectrum for velocity fluctuations

$$
E_{K K}(n)=C_{K} \Pi_{\epsilon}^{2 / 3} n^{-5 / 3}
$$

where $C_{Z}=0.2$ and $C_{K}=6$ are taken to be estimated of universal constants and $\beta$ is estimated at midlatitude (radius) for planetary (laboratory) flow (see in supporting information section S1) (Galperin et al., 2010; Sukoriansky et al., 2002). The indices $n$ are nondimensional total wavenumbers. For a given $n$, a typical length scale $L$ is given by $L=\alpha_{m n} / n$ in cylindrical geometry, where $\alpha_{m n}$ are zeros of the Bessel functions and $m$ are zonal indices, and by $L=2 \pi R / n$ when spherical harmonic functions are invoked in planetary geometry, where $R$ is the planetary radius (spectral analysis is detailed in supporting information section $\mathrm{S} 1$ ). The zonal and KK spectra intersect at the scale $L_{\beta}$, which corresponds to the transition scale beyond which planetary vorticity preferentially channels energy into the zonal direction, favoring Rossby waves.

As all other parameters are intrinsic properties of the system (i.e., of the laboratory experiment or the planets), the energy transfer rate $\Pi_{\epsilon}$ is the only free parameter when setting $L_{\beta}$. To estimate $\Pi_{\epsilon}$ we fit the KK-law (Equation 3) with the spectrum of velocity fluctuations, shown as dotted and solid black lines in Figure 1. The range of wave numbers where the fit applies appears to be small in the laboratory, well defined for Jupiter observations, and slightly distorted by an energetic bump in the Saturn GCM. The robustness of our approach is ensured by an independent estimate of the energy transfer rate $\Pi_{\epsilon}$ using energy fluxes. Thus, we fit the KK-law spectra with a value of $\Pi_{\epsilon}$ that both complies with our estimate from the energy fluxes and a subrange in the spectra that shows a -(5/3) slope. Note that in all cases shown in Figure 1, the wide range of negative energy fluxes show the existence of upscale energy transfers that sustain the jets.

Here, for Jupiter we find $\Pi_{\epsilon}=9 \times 10^{-5} \mathrm{~W} \mathrm{~kg}^{-1}$ using our fit of the energy spectrum. This estimate is consistent with the energy flux magnitude for Jupiter, which reaches the minimum (negative) value around $\Pi_{\epsilon} \sim 5 \times 10^{-5} \mathrm{~W} \mathrm{~kg}^{-1}$, and with the range $10^{-5} \leq \Pi_{\epsilon} \leq 10^{-4} \mathrm{~W} \mathrm{~kg}^{-1}$ from Galperin, Young, et al. (2014) and Young and Read (2017). For the Saturn GCM, we find $\Pi_{\epsilon}=0.13 \times 10^{-5} \mathrm{~W} \mathrm{~kg}^{-1}$, consistent with the minimum 
Table 1

Summary of the Measured Diagnostics in All Data Sets

\begin{tabular}{|c|c|c|c|c|c|}
\hline & Data set & $\begin{array}{c}\Pi_{\epsilon} \\
\left(\mathrm{W} \mathrm{kg}^{-1}\right)\end{array}$ & $\begin{array}{c}L_{M} \\
(\mathrm{~cm}) \text { or }(\mathrm{km})\end{array}$ & $\begin{array}{c}L_{\beta} \\
(\mathrm{cm} \text { or } \mathrm{km})\end{array}$ & $L_{M} / L_{\beta}$ \\
\hline \multirow[t]{9}{*}{ Laboratory } & 1: Westward jet $90^{\circ}$ & $(2.1-0.7) \times 10^{-8}$ & $1.38( \pm 0.7)$ & $2.04( \pm 0.3)$ & $0.68( \pm 0.36)$ \\
\hline & 2: Westward jet $90^{\circ}$ & $(11-13) \times 10^{-8}$ & $1.80( \pm 0.9)$ & $3.14( \pm 0.1)$ & $0.57( \pm 0.29)$ \\
\hline & 3: Westward jet $90^{\circ}$ & $(29-7.0) \times 10^{-8}$ & $1.90( \pm 1.1)$ & $3.26( \pm 0.6)$ & $0.58( \pm 0.36)$ \\
\hline & 1: Eastward jet $90^{\circ}$ & $(2.1-1.5) \times 10^{-8}$ & $1.00( \pm 0.7)$ & $2.15( \pm 0.1)$ & $0.46( \pm 0.32)$ \\
\hline & 2: Eastward jet $90^{\circ}$ & $(11-12) \times 10^{-8}$ & $1.87( \pm 1.0)$ & $3.12( \pm 0.04)$ & $0.60( \pm 0.32)$ \\
\hline & 3: Eastward jet $90^{\circ}$ & $(21-40) \times 10^{-8}$ & $2.40( \pm 1.0)$ & $3.73( \pm 0.3)$ & $0.64( \pm 0.27)$ \\
\hline & 1: Westward jet $180^{\circ}$ & $(2.0-0.6) \times 10^{-8}$ & $1.25( \pm 0.7)$ & $2.00( \pm 0.3)$ & $0.62( \pm 0.36)$ \\
\hline & 2: Westward jet $180^{\circ}$ & $(8.0-3.0) \times 10^{-8}$ & $1.64( \pm 1.0)$ & $2.65( \pm 0.4)$ & $0.62( \pm 0.38)$ \\
\hline & 3: Westward jet $180^{\circ}$ & $(20-14) \times 10^{-8}$ & $1.89( \pm 1.1)$ & $3.34( \pm 0.1)$ & $0.56( \pm 0.33)$ \\
\hline \multirow[t]{3}{*}{ Jupiter } & 2-D Cassini maps & $(9-5) \times 10^{-5}$ & $5,200( \pm 2,500)$ & $11,600( \pm 970)$ & $0.45( \pm 0.22)$ \\
\hline & QGPV: CIRS - IRIS & $\sim(0.3-2) \times 10^{-5}$ & $3,500-5,000$ & $6,000-8,600$ & 0.58 \\
\hline & IPV: CIRS - IRIS & $\sim(0.6-3) \times 10^{-5}$ & $4,200-5,600$ & $7,200-9,700$ & 0.58 \\
\hline \multirow[t]{3}{*}{ Saturn } & Global Climate Model & $(0.13-0.25) \times 10^{-5}$ & $3,400( \pm 2,000)$ & $5,200( \pm 490)$ & $0.64( \pm 0.38)$ \\
\hline & QGPV & $\sim 0.9 \times 10^{-5}$ & 4,200 & 7,200 & 0.58 \\
\hline & IPV & $\sim 0.5 \times 10^{-5}$ & 3,700 & 6,400 & 0.58 \\
\hline
\end{tabular}

Note. Details for each laboratory configuration are listed in supporting information Table S1. The turbulent power $\Pi_{\epsilon}$ reported in this table and used to estimate $L_{\beta}$ are obtained using a fit to the velocity fluctuations spectra and spectral energy fluxes and are in $\mathrm{W} \mathrm{kg}^{-1}$. Typical length scales $L_{M}$ and $L_{\beta}$ are in $\mathrm{cm}$ in the laboratory and in $\mathrm{km}$ for Jupiter and Saturn, respectively. Estimates in red are obtained using the averaged relationship with its averaged standard deviation $L_{M} / L_{\beta} \simeq 0.58 \pm 0.3$. In the laboratory one can also consider the standard error of the mean for the relationship $L_{M} / L_{\beta} \simeq 0.59 \pm 0.02$. Typical length scales and the ratio $L_{M} / L_{\beta}$ are reported with their standard deviation values in brackets, \pm 0.3 is the averaged value over the three data sets, and \pm 0.02 is the standard error of the mean over the nine experimental runs in the laboratory. For QGPV and IPV measurements the standard deviation is $\pm 2,000 \mathrm{~km}$ (note reported in the table). The averaging procedures and the computation of the standard deviations are detailed in supporting information section S1. Values in red are all quantities that are calculated using the averaged ratio $L_{M} / L_{\beta}=0.58$ (in red itself). Black values instead lead to the calculation of the averaged ratio $L_{M} / L_{\beta}$.

(negative) value of the energy flux $\Pi_{\epsilon} \sim 0.25 \times 10^{-5} \mathrm{~W} \mathrm{~kg}^{-1}$ (see also Cabanes et al., 2020). This estimate of $\Pi_{\epsilon}$ is much lower than for Jupiter but is likely to be underestimated as the model does not include several important energy sources such as moist convection and turbulent instabilities caused by an internal heat flux. Numerical approximations also damp the global energy budget by implementing an artificial hyperdiffusivity that compensates for unresolved subgrid-scale processes and substantially reduces turbulent mixing at the smallest scales (Cabanes et al., 2020). In the laboratory, the westward jet presented in Figure 1 has an energy transfer rate $\Pi_{\epsilon}=20 \times 10^{-8} \mathrm{~W} \mathrm{~kg}^{-1}$, consistent with the minimum (negative) value of the energy flux $\Pi_{\epsilon} \sim 14 \times 10^{-8} \mathrm{~W} \mathrm{~kg}^{-1}$. In the data set of laboratory experiments, the energy transfer rate values for $\Pi_{\epsilon}$ vary between $(2-30) \times 10^{-8} \mathrm{~W} \mathrm{~kg}^{-1}$ using our fit of the energy spectra and between $(0.6-40) \times 10^{-8} \mathrm{~W} \mathrm{~kg}^{-1}$ using the minimum value from spectral energy fluxes (see supporting information figures for additional spectra and energy fluxes). It has been established elsewhere that structure function analyses also confirm our estimate of the energy transfer rate $\Pi_{\epsilon}$ and corroborate the robustness of our estimates of $L_{\beta}$ (Galperin et al., 2016, for laboratory jets, and Young \& Read, 2017, for Jupiter). For all data sets, we compute $L_{\beta}$ using the energy transfer rates $\Pi_{\epsilon}$ estimated from both the energy fluxes and our fit of the energy spectra. All values of $\Pi_{\epsilon}$ are reported in Table 1 together with the averaged estimate of $L_{\beta}$ and its standard deviation. In all three cases, laboratory, Jupiter, and Saturn, our estimate of the theoretical scale $L_{\beta}$ successfully fits the intersection of the zonal and residual spectra.

Figure 2 displays sample profiles of instantaneous mean zonal velocity, their corresponding PV profiles, and their associated monotonized PV profiles computed using a Thorpe-like sorting algorithm, for all three cases. In all profiles, PV is strongly mixed and homogenized on jet flanks by the turbulent eddies. This reshapes the large-scale PV distribution into a staircase, causing velocity profiles to sharpen. According to the classical understanding, jets form into bands of monotonic PV, as claimed by Marcus and Lee (1998), 

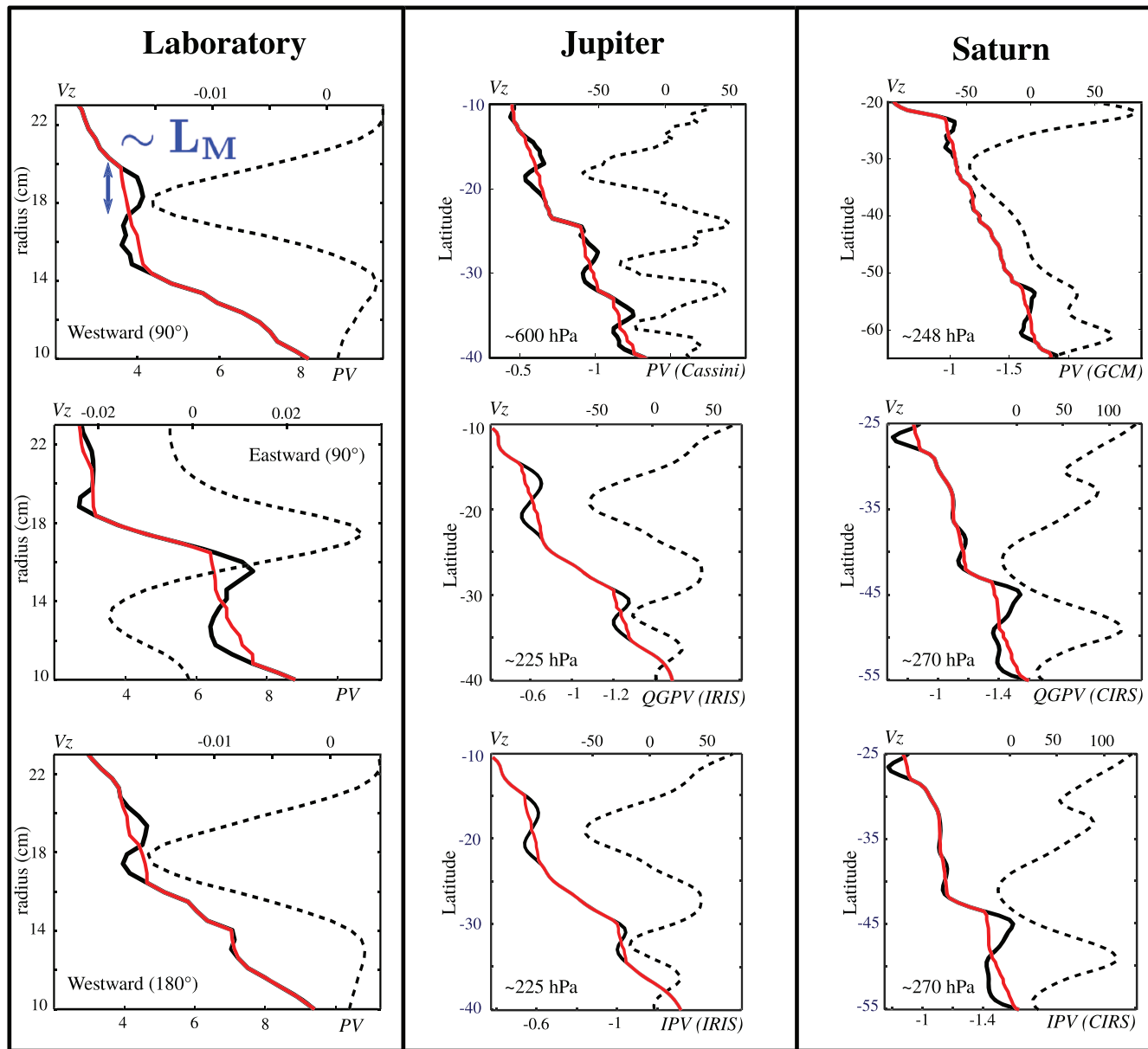

Figure 2. Profiles of the zonal velocity and the corresponding potential vorticity. Instantaneous zonally averaged zonal velocity profiles are dashed lines in $\mathrm{m} \mathrm{s}^{-1}$. Instantaneous nondimensional (normalized by the rotation rate $\Omega$ and the mean layer depth) potential vorticity (PV), isentropic potential vorticity (IPV), and quasi-geostrophic potential vorticity (QGPV) profiles are solid black lines. In blue is an indicative typical scale $\mathrm{L}_{\mathrm{M}}$. The names in brackets indicate where the data come from: IRIS stands for InfraRed Interferometric Spectrometer (NASA Voyager), and CIRS stands for Cassini Composite InfraRed Spectrometer (NASA Cassini), which delivered thermal measurements. Monotonized potential vorticity (using a sorting algorithm, see supporting information section $\sim \mathrm{S} 1$ ) are in red solid lines. Atmospheric pressure levels at which PV is measured are labeled in each panel.

Dritschel and McIntyre (2008), and Marcus and Shetty (2011). However, we clearly show Figure 2 that staircase features are actually strongly nonmonotonic (also see supporting information figures of PV profiles for experiments, observations, and simulation). We suggest that this nonmonotonicity contains information about rich dynamics involving energy exchanges between jets, Rossby waves, and turbulent eddies and that the (average) magnitude of these energy exchanges can be summarized in the turbulent power $\Pi_{\epsilon}$.

PV profiles are nonmonotonic over a typical length scale $L_{M}$, as indicated in Figure 2. We suggest that $L_{M}$ can be interpreted as the latitudinal distance over which PV is transported by turbulent mixing but which is then limited by planetary vorticity gradients from converting turbulent eddies into zonal jets. $L_{M}$ is nearly equivalent to $L_{\beta}$, which defines the scale above which the jets become the most energetic scale of motions, that is, where $E_{Z}>E_{K K}$.

To extract the length scales $L_{M}$, we monotonize the latitudinal PV, IPV, and QGPV profiles using the sorting algorithm. To obtain an overall characterization of these turbulent processes, we consider the root-mean-square (RMS) and the standard deviation for $L_{M}$ over all longitudes, latitudes, and times (the monotonization and averaging procedure are described in supporting information section S1). These scales $L_{M}$ are summarized in Table 1 . They correspond to 1-2 $\pm 0.9 \mathrm{~cm}$ in the laboratory and to thousands of kilometers in the gas giants: $\sim 4,500 \mathrm{~km}$ for Jupiter and $\sim 3,800 \mathrm{~km}$ for Saturn and with standard deviations 


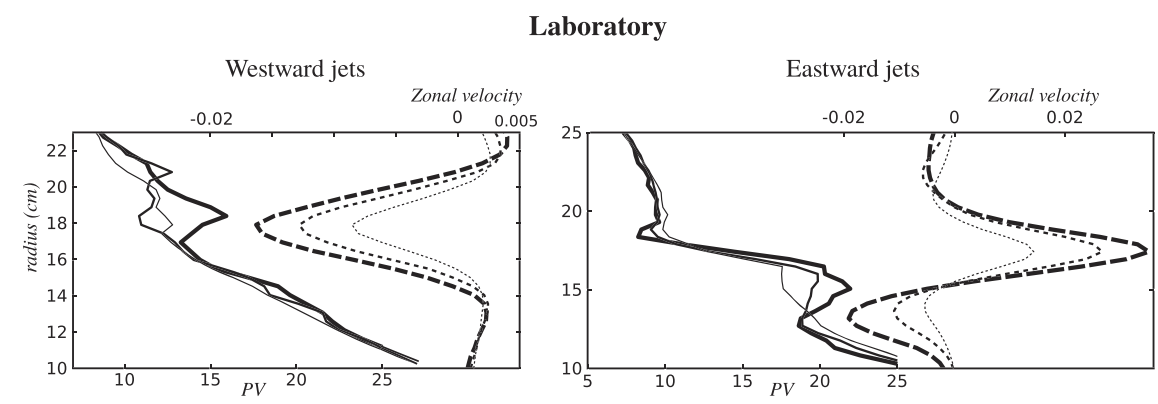

Figure 3. Laboratory potential vorticity and averaged zonal velocity. PV profiles are thick lines in units of $\times 10^{-4} \mathrm{~s}^{-1}$, and zonal velocity profiles are dashed lines in $\mathrm{m} \mathrm{s}^{-1}$. The left (right) panels are three westward (eastward) jets for a $180^{\circ}\left(90^{\circ}\right)$ arc of magnets and for three different forcing currents $I=2,4$, and $6 \mathrm{~A}$, indicated by the lines' thickness and referenced as Data Sets 1, 2, and 3 in Table 1. Increasing thickness means increasing current intensity, hence increasing forcing strength and nonmonotonicity of the PV profiles.

of $\pm 2,000 \mathrm{~km}$. Overall, the relationship between $L_{M}$ and $L_{\beta}$ can be summarized as $L_{M} / L_{\beta} \simeq 0.58 \pm 0.3 ; L_{M}$ is an intermediate length scale between the small-scale energy sources and $L_{\beta}$. This relationship holds reasonably well across all three data sets (laboratory, Jupiter, and Saturn), but the uncertainty calls for further analysis of a local estimate, that is, at smaller scale, rather than a global estimate of the turbulent energy transfers $\Pi_{\epsilon}$.

From our laboratory experiments, we show in Figure 3 that, as $\Pi_{\epsilon}$ increases, the nonmonotonicity of the PV profiles also increases. This confirms the existence of a common trend between $L_{M}$ and $L_{\beta}$ and supports our use of PV monotonization to characterize the turbulent power in the flow. We can also compute the standard error of the mean over the nine experimental runs, leading to the ratio with its uncertainty $L_{M} / L_{\beta} \simeq 0.59 \pm 0.02$, instead of the mean of the standard deviation, leading to the previous ratio $L_{M} / L_{\beta} \simeq 0.58 \pm 0.3$.

Here, one has to consider that the reproducibility of the ratio over the nine experimental runs reduces the uncertainty in the particular configuration of the laboratory. We note, however, that IPV and QGPV diagnostics for Jupiter lead to values of $L_{M}$ (thus values of $\Pi_{\epsilon}$ ) that are slightly lower than those obtained using PV profiles from the Cassini 2-D horizontal vorticity fields (Table 1). This is expected, as IPV and QGPV are derived from zonally averaged velocity and temperature measurements, in which the averaging procedure likely reduces the turbulent signature in the PV profiles. Finally, the Saturn GCM value for $L_{M}$ is also low, due to numerical limitations when simulating highly turbulent flow, as described above.

\section{Implications for Planetary Turbulence}

We have demonstrated that, for rotating turbulence with a $\beta$ effect and an upscale energy cascade, there exists a length scale $L_{M}$ which provides an overall picture of the dynamics by allowing for a straightforward estimate of the intensity of turbulent energy transfer $\Pi_{\epsilon}$. This estimate relies on the relationship $L_{M} / L_{\beta} \simeq 0.58 \pm 0.3$, which we argue is universal by the unprecedented set of data to which it applies: laboratory experiments, GCMs, and direct observations of two different planetary atmospheres. Also, the independent procedures used to estimate $L_{\beta}$ (i.e., using a fit of the energy spectra and spectral energy fluxes) argue for the universality of the relationship $L_{M} / L_{\beta} . L_{M}$ can be computed easily just from a zonal mean zonal velocity profile, the planetary rotation rate, and an estimate of the atmospheric scale height, which is much more amenable than the 2-D horizontal wind maps required hitherto.

With $\Pi_{\epsilon}$, one can retrieve the global distribution of kinetic energy, from small-scale sources up to the jet scale, by using the theoretical energy spectra $E_{Z}(n)$ and $E_{K K}(n)$. In flows with spatially inhomogeneous energy sources, this method has the power to trace back the turbulent energetic sources in planetary atmospheres, which is otherwise impractical. The theory applies in several natural settings, such as atmospheres of the gas giants and of exoplanets as well as the Earth's oceans.

For Saturn, where $\Pi_{\epsilon}$ has not yet been measured, we can use $L_{M}$ to predict its value. By monotonizing IPV and QGPV profiles from Read et al. (2009) and then using $L_{M} / L_{\beta} \simeq 0.56 \pm 0.3$, we find for Saturn's atmosphere a turbulent power of $0.5 \times 10^{-5} \leq \Pi_{\epsilon} \leq 0.9 \times 10^{-5} \mathrm{~W} \mathrm{~kg}^{-1}$, with an uncertainty of 1 order of 
magnitude that corresponds to $L_{M} \simeq 3,700$ to 4,200 $( \pm 2,000) \mathrm{km}$ (red values in Table 1$)$. As discussed above, this estimate of $\Pi_{\epsilon}$ is likely to be an underestimate based on the averaged QGPV and IPV from which it is estimated. Also, measuring $\Pi_{\epsilon}$ offers the opportunity to estimate the latitudinal eddy diffusivity coefficient, $K_{\theta}$, within Jupiter's and Saturn's atmospheres. $K_{\theta}$ reflects the turbulent mixing in the meridional direction (along the latitude), and we find $K_{\theta} \sim(1-7) \times 10^{6} \mathrm{~m}^{2} \mathrm{~s}^{-1}$ for Jupiter and $\sim(1.1-1.6) \times 10^{6} \mathrm{~m}^{2} \mathrm{~s}^{-1}$ for Saturn (see supporting information section $\mathrm{S} 1$ ). Using these eddy diffusivity coefficients with a one-dimensional diffusion equation, one can predict the meridional dispersion of any natural conservative tracer in these atmospheres. Our results are in good agreement with the values obtained by Friedson et al. (1999) from the observation of the meridional spread of comet Shoemaker-Levy 9's debris for Jupiter and dispersion of gases for Saturn (Friedson \& Moses, 2012). We conclude that Saturn's atmosphere is likely to have an intensity of turbulent energy transfer $\left(\Pi_{\epsilon}\right)$ and thus an intensity of turbulent mixing $\left(K_{\theta}\right), 2$ to 4 times less than Jupiter's. We can speculate that part of this difference simply reflects the fact that Saturn is nearly twice as far from the Sun as Jupiter and hence receives a quarter of the solar energy input. Nonetheless, our estimate of Saturn's turbulent power carries an uncertainty that largely exceeds a factor 4 (with a 1 order of magnitude uncertainty). Such uncertainty likely reflects that the global atmospheric dynamics is summarized in a single value of energy transfer rate $\Pi_{\epsilon}$ while the parameter $\beta$ itself is latitudinally dependent. However, this is the first estimate of Saturn's turbulent power using the available data, which was not designed for such an analysis, and this study paves the way for future data collection from planetary atmospheres.

Because it is universal, the relationship between $L_{M}$ and $L_{\beta}$ can be used to diagnose the intensity of turbulent energy transfer (as well as turbulent mixing) in many other natural settings for which PV is conserved, such as the Earth's ocean and newly discovered exoplanets.

\section{Data Availability Statement}

Data sets related to this article can be found at https://doi.org/10.5281/zenodo.3634814, an open-source online data repository hosted at Zenodo (Zenodo.org).

\section{References}

This project has received funding from the European Union's Horizon 2020 research and innovation programme under the Marie Skodowska-Curie grant agreement $\mathrm{N}^{\circ}$ 797012. B. G. was partially supported by the NASA/NOAA Ocean Surface Topography Science Team (Grant Nos. 2500171500 and 2500171600 ) and by a University of South Florida Nexus Initiative (UNI) Award (No. RP0101). R. M. B. Y. and P. L. R. acknowledge funding support from the UK Science and Technology Facilities Council (STFC) under Grants ST/K502236/1, ST/K00106X/1, and ST/I001948/1.

Boffetta, G., \& Musacchio, S. (2010). Evidence for the double cascade scenario in two-dimensional turbulence. Physical Review E, 82(1),
Arbic, B. K., Müller, M., Richman, J. G., Shriver, J. F., Morten, A. J., Scott, R. B., et al. (2014). Geostrophic turbulence in the frequency-wavenumber domain: Eddy-driven low-frequency variability. Journal of Physical Oceanography, 44(8), $2050-2069$. Augier, P., \& Lindborg, E. (2013). A new formulation of the spectral energy budget of the atmosphere, with application to two high-resolution general circulation models. Journal of the Atmospheric Sciences, 70(7), 2293-2308.

Boer, G. J. (1983). Homogeneous and isotropic turbulence on the sphere. Journal of the Atmospheric Sciences, 40(1), 154-163. 016307.

Cabanes, S., Spiga, A., \& Young, R. M. B. (2020). Global climate modeling of Saturn's atmosphere. Part III: Global statistical picture of zonostrophic turbulence in high-resolution 3D-turbulent simulations. Icarus, 345, 113705. https://doi.org/10.1016/j.icarus.2020.113705

Chen, S., Ecke, R. E., Eyink, G. L., Rivera, M., Wan, M., \& Xiao, Z. (2006). Physical mechanism of the two-dimensional inverse energy cascade. Physical Review Letters, 96(8), 084502.

Cho, J. Y. K., \& Polvani, L. M. (1996). The morphogenesis of bands and zonal winds in the atmospheres on the giant outer planets. Science, 273(5273), 335.

Clayson, C. A., \& Kantha, L. (2008). On turbulence and mixing in the free atmosphere inferred from high-resolution soundings. Journal of Atmospheric and Oceanic Technology, 25(6), 833-852.

Dritschel, D. G., \& McIntyre, M. E. (2008). Multiple jets as PV staircases: The Phillips effect and the resilience of eddy-transport barriers. Journal of the Atmospheric Sciences, 65(3), 855-874.

Dubos, T., Dubey, S., Tort, M., Mittal, R., Meurdesoif, Y., \& Hourdin, F. (2015). DYNAMICO-1.0, an icosahedral hydrostatic dynamical core designed for consistency and versatility. Geoscientific Model Development, 8(10), 3131-3150.

Ertel, H., \& Rossby, C.-G. (1949). A new conservation-theorem of hydrodynamics. Geofisica Pura e Applicata, 14(3-4), 189-193.

Friedson, A. J., \& Moses, J. I. (2012). General circulation and transport in Saturn's upper troposphere and stratosphere. Icarus, 218(2), 861-875.

Friedson, A. J., West, R. A., Hronek, A. K., Larsen, N. A., \& Dalal, N. (1999). Transport and mixing in Jupiter's stratosphere inferred from comet S-L9 dust migration. Icarus, 138(1), 141-156.

Galperin, B., Hoemann, J., Espa, S., \& Di Nitto, G. (2014). Anisotropic turbulence and Rossby waves in an easterly jet: An experimental study. Geophysical Research Letters, 41, 6237-6243. https://doi.org/10.1002/2014GL060767

Galperin, B., Hoemann, J., Espa, S., Di Nitto, G., \& Lacorata, G. (2016). Anisotropic macroturbulence and diffusion associated with a westward zonal jet: From laboratory to planetary atmospheres and oceans. Physical Review E, 94(6), 063102.

Galperin, B., Sukoriansky, S., \& Dikovskaya, N. (2010). Geophysical flows with anisotropic turbulence and dispersive waves: flows with a $\beta$-effect. Ocean Dynamics, 60(2), 427-441.

Galperin, B., Young, R. M. B., Sukoriansky, S., Dikovskaya, N., Read, P. L., Lancaster, A. J., \& Armstrong, D. (2014). Cassini observations reveal a regime of zonostrophic macroturbulence on Jupiter. Icarus, 229, 295-320.

Gargett, A., \& Garner, T. (2008). Determining Thorpe scales from ship-lowered CTD density profiles. Journal of Atmospheric and Oceanic Technology, 25(9), 1657-1670. 
Gavrilov, N. M., Luce, H., Crochet, M., Dalaudier, F., \& Fukao, S. (2005). Turbulence parameter estimations from high-resolution balloon temperature measurements of the MUTSI-2000 campaign. Annales Geophysicae, 23(7), 2401-2413.

Gierasch, P. J., Conrath, B. J., \& Read, P. L. (2004). Nonconservation of Ertel potential vorticity in hydrogen atmospheres. Journal of the Atmospheric Sciences, 61(15), 1953-1965.

Guerlet, S., Spiga, A., Sylvestre, M., Indurain, M., Fouchet, T., Leconte, J., et al. (2014). Global climate modeling of Saturn's atmosphere. Part I: Evaluation of the radiative transfer model. Icarus, 238, 110-124.

Ingersoll, A. P., \& Pollard, D. (1982). Motion in the interiors and atmospheres of Jupiter and Saturn: Scale analysis, anelastic equations, barotropic stability criterion. Icarus, 52(1), 62-80.

Kantha, L., \& Hocking, W. (2011). Dissipation rates of turbulence kinetic energy in the free atmosphere: MST radar and radiosondes. Journal of Atmospheric and Solar-Terrestrial Physics, 73(9), 1043-1051.

Klymak, J. M., \& Legg, S. M. (2010). A simple mixing scheme for models that resolve breaking internal waves. Ocean Modelling, 33(3-4), 224-234.

Kraichnan, R. H. (1967). Inertial ranges in two dimensional turbulence. Physics of Fluids, 10(7), 1417-1423.

Marcus, P. S., \& Lee, C. (1998). A model for eastward and westward jets in laboratory experiments and planetary atmospheres. Physics of Fluids, 10(6), 1474-1489.

Marcus, P. S., \& Shetty, S. (2011). Jupiter's zonal winds: Are they bands of homogenized potential vorticity organized as a monotonic staircase? Philosophical Transactions of the Royal Society A: Mathematical, Physical and Engineering Sciences, 369(1937), 771-795. Pedlosky, J. (2013). Geophysical fluid dynamics: Springer Science \& Business Media.

Phillips, N. A. (1956). The general circulation of the atmosphere: A numerical experiment. Quarterly Journal of the Royal Meteorological Society, 82(352), 123-164.

Read, P. L., Conrath, B. J., Fletcher, L. N., Gierasch, P. J., Simon-Miller, A. A., \& Zuchowski, L. C. (2009). Mapping potential vorticity dynamics on Saturn: Zonal mean circulation from Cassini and Voyager data. Planetary and Space Science, 57(14-15), 1682-1698.

Read, P. L., Gierasch, P. J., Conrath, B. J., Simon-Miller, A., Fouchet, T., \& Yamazaki, Y. H. (2006). Mapping potential-vorticity dynamics on Jupiter. I: Zonal-mean circulation from Cassini and Voyager 1 data. Quarterly Journal of the Royal Meteorological Society, 132(618), 1577-1603.

Read, P. L., Tabataba-Vakili, F., Wang, Y., Augier, P., Lindborg, E., Valeanu, A., \& Young, R. M. B. (2018). Comparative terrestrial atmospheric circulation regimes in simplified global circulation models. Part II: Energy budgets and spectral transfers. Quarterly Journal of the Royal Meteorological Society, 144(717), 2558-2576.

Rhines, P. B. (1975). Waves and turbulence on a beta-plane. Journal of Fluid Mechanics, 69(03), 417-443.

Schneider, T., \& Liu, J. (2009). Formation of jets and equatorial superrotation on Jupiter. Journal of the Atmospheric Sciences, 66(3), 579-601.

Spiga, A., Guerlet, S., Millour, E., Indurain, M., Meurdesoif, Y., Cabanes, S., et al. (2020). Global climate modeling of Saturn's atmosphere. Part II: Multi-annual high-resolution dynamical simulations. Icarus, 335, 113377.

Sukoriansky, S., Galperin, B., \& Dikovskaya, N. (2002). Universal spectrum of two-dimensional turbulence on a rotating sphere and some basic features of atmospheric circulation on giant planets. Physical Review Letters, 89(12), 124501.

Thorpe, S. A. (2005). The turbulent ocean: Cambridge University Press.

Vallis, G. K. (2006). Atmospheric and oceanic fluid dynamics: Fundamentals and large-scale circulation: Cambridge University Press.

Vallis, G. K., \& Maltrud, M. E. (1993). Generation of mean flows and jets on a beta plane and over topography. Journal of Physical Oceanography, 23(7), 1346-1362.

Young, R. M. B., \& Read, P. L. (2017). Forward and inverse kinetic energy cascades in Jupiter's turbulent weather layer. Nature Physics, 13(11), 1135-1140. 